Abstract FRI0761-HPR - Table 1. Individual- and contextual-dependent variables

\begin{tabular}{|c|c|c|c|c|c|c|c|c|}
\hline & & \multicolumn{5}{|c|}{ Random Effects Pooled Prevalence } & \multicolumn{2}{|c|}{ Univariate Meta-regression } \\
\hline & & Studies & Estimates & Pooled Estimate & $95 \% \mathrm{Cl}$ & $\mathrm{I}^{2}$ & $1^{2}$ & $\mathrm{p}$-value \\
\hline \multirow[t]{2}{*}{ Gender } & Males ( $n=242808)$ & 11 & 11 & 6.7 & $4.5-8.8$ & 98.6 & 98.8 & $<0.01$ \\
\hline & Females $(n=290395)$ & 11 & 11 & 10.9 & $8.1-13.7$ & 98.7 & & \\
\hline \multirow[t]{2}{*}{ WHO Geographical Region } & Europe $(n=595876)$ & 17 & 21 & 9.1 & $7.1-11.2$ & 99.8 & 99.7 & 0.208 \\
\hline & Americans $(n=16611)$ & 6 & 6 & 10.9 & $5.1-16.7$ & 99.4 & & \\
\hline \multirow[t]{2}{*}{ Latitude } & $<37^{\circ}(\mathrm{n}=6651)$ & 6 & 6 & 10.5 & $4.5-16.5$ & 98.9 & 99.8 & 0.622 \\
\hline & $>37^{\circ}(n=612233)$ & 19 & 23 & 9.2 & $7.3-11.1$ & 99.8 & & \\
\hline \multirow[t]{2}{*}{ Survey Type } & Personal $(n=551483)$ & 18 & 22 & 10.0 & $7.7-12.3$ & 99.5 & 99.5 & 0.746 \\
\hline & Non-Personal $(n=67401)$ & 7 & 7 & 7.8 & $4.8-10.7$ & 99.4 & & \\
\hline \multirow[t]{2}{*}{ WHO Development Status } & Developed $(\mathrm{n}=611999)$ & 21 & 25 & 8.7 & $7.0-10.5$ & 99.7 & 99.7 & 0.084 \\
\hline & Developing $(\mathrm{n}=6885)$ & 4 & 4 & 13.9 & $6.4-21.4$ & 98.5 & & \\
\hline \multirow[t]{2}{*}{ Socioeconomic Measures } & GINI Index (n=618884) & 25 & 29 & & & & 99.5 & 0.258 \\
\hline & HDI $(n=618884)$ & 25 & 29 & & & & 99.4 & 0.002 \\
\hline \multirow[t]{2}{*}{ Reporting Quality } & High $(n=583819)$ & 17 & 21 & 10.0 & $7.5-12.5$ & 99.8 & 99.7 & 0.237 \\
\hline & Low $(n=583819)$ & 8 & 8 & 8.0 & $5.8-10.2$ & 98.4 & & \\
\hline
\end{tabular}

Key: "Studies" refer to number of manuscripts, while "Estimates" relate to the number of countries within a manuscript that provided CWP prevalence estimates.

of socioeconomic position), GINI coefficient (measure of inequality), and latitude were added. Methodological reporting quality was assessed using the STROBE checklist [1]. Meta-analyses were conducted to determine global CWP prevalence, and individual- and contextual-dependent factors were evaluated using metaregression analyses.

Results: Thirty studies across 20 countries (630,654 participants) met the inclusion criteria. Studies varied in CWP diagnostic criteria, survey method, and reporting quality. Study CWP sample prevalence ranged from 1.4-24.0\%, with CWP in men ranging from 1.1-15.3\%, while in women it ranged from $1.7-22.1 \%$. Estimated overall CWP prevalence was $9.4 \%$ (7.6-11.3\%). By gender, the global CWP prevalence estimate in women was significantly higher than for men $(10.9 \%$ [8.1-13.7\%] v 6.7\% [4.5-8.8\%], $\mathrm{p} \leq 0.01$; Table). A meta-regression of the contextual factors showed the HDI was related to CWP prevalence $(p=0.002)$, while other measures such as survey type, methodological reporting quality and GINI index showed no significant effect.

Conclusions: Globally CWP affects one in ten individuals within the general population, with women more likely to experience CWP than men. HDI was noted to be the socioeconomic factor related to CWP prevalence, with those in more developed countries having a lower CWP prevalence than those in less developed countries. There was a lack of data from countries with a lower socioeconomic position, and further CWP data in these areas can help determine if there are socioeconomic effects associated with CWP prevalence and can refine the CWP prevalence estimate.

References:

[1] Poorolajal, J., et al., Epidemiology and health, 2011. 33.

Disclosure of Interest: None declared

DOI: 10.1136/annrheumdis-2017-eular.3638

\section{FRI0762-HPR PREVALENCE OF TOBACCO USE IN AUTOIMMUNE INFLAMMATORY RHEUMATIC DISEASES (AIRDS): EFFICACY OF COUNSELING IN QUITTING TOBACCO USE}

S.S. Baghel, R. Rawat, R.D. Thakran, C. Messi, S. Kapoor, S. Garg, V. Kashyap, Q. Zaheer, A.N. Malaviya. Rheumatology, Indian Spinal Injuries Centre, New Delhi, India

Background: Autoimmune inflammatory rheumatic diseases (AIRDs) are chronic in nature and common presentation involves the musculoskeletal system. Off lately, the researches have proved the strong association of tobacco in the development of the disease and increasing severity as well as reducing treatment efficacy. Therefore, awareness about the deleterious effect of tobacco and counselling to quit the same should be an imperative part of the patient education. Objectives: To assess the prevalence of tobacco use among patients with AIRDs and the effect of counselling on quitting tobacco.

Methods: AIRDs patients attending the rheumatology clinic, willing to participate in the survey were enrolled in this study. Tobacco use was categorized as active [smoking or oral tobacco use] or passive (when the patient was exposed to tobacco smoke).All the information collected in pre-design form, which included demographic information and current status of tobacco use. They were also counselled about the methods of quitting tobacco and its importance and short-term follow-up details were recorded.

Results: 361 patients were enrolled in this study, out of them 162 (44.87\%) were tobacco-naive and 199 patients $(55.12 \%)$ were either active or passive tobacco users, among them active tobacco user $113(56.78 \%)$, and passive user 86 (43.21\%).Active tobacco users were further classified into mild 53 $(47 \%)$, moderate $50(44 \%)$ and severe 10 (9\%) users. Other characteristics were as follows; Female: Male ratio1.02:1 [F: M (183:178)], Rheumatoid arthritis 268 (74.2\%) [F: M (171:97)], Spondyloarthropathy 93 (25.8\%) [M: F (81:12)]. Residential details were: urban $284(79 \%)$ and rural $79(21 \%)$. Educational status: $28(8 \%)$ illiterate, 148 (41\%) basic education, $106(29 \%)$ graduate, $79(22 \%)$ postgraduate. Co-morbidities status: 123 (34\%) having multiple co-morbidities and $238(66 \%)$ have no co-morbidities.

$276(75 \%)$ patients were unaware of the ill effects of tobacco use in AIRDs whereas, remaining 85 (25\%) were having little knowledge about it.

The results of persistent counselling and creating awareness with the help of audio-visual aids were $49 / 113(43.4 \%)$ quit from the active user's category, whereas, 64/113 (56.6\%) were unable to quit. Among passive users, 9/86 $(10.46 \%)$ could able to quit whereas in a large significant number of patients follow-up information could not be obtained.

Conclusions: The use of tobacco was significantly higher in males $(61.80 \%)$ than females (38.19\%).But females were mainly passively exposed to tobacco. By an intense and regular counselling by the specialist rheumatology nurses; $43.4 \%$ of active and $10.4 \%$, passive users were able to quit tobacco.

\section{References:}

[1] Aimer P, Treharne GJ, Stebbings S, Frampton C, Cameron V, Kirby S et al. Efficacy of a Rheumatoid Arthritis -Specific Smoking Cessation Program: A Randomized Controlled Pilot Trial. Arthritis Care Res 2017; 69:28-37.

[2] Naranjo A, Khan NA, Cutolo M, Lee SS, Lazovskis J, Pandya S, et.al. Smoking cessation advice by rheumatologist: results of an international survey on behalf of the QUEST-RA group.Rheumatology (oxford) 2014; 53:1825-29.

Acknowledgements: The author also wants to thanks, Mr Himanshu Negi (Data Entry help).

Disclosure of Interest: S. Baghel: None declared, R. Rawat: None declared, R. Thakran: None declared, C. Messi: None declared, S. Kapoor Consultant for: Advisory board of Novartis,Pfizer, S. Garg Consultant for: Advisory board of Intas, V. Kashyap: None declared, Q. Zaheer: None declared, A. Malaviya Consultant for: Advisory board of IPCA, Janssen, Pfizer, Roche, BMS,Dr. Reddy's, Zydus DOI: 10.1136/annrheumdis-2017-eular.2089

\section{FRI0763-HPR INVESTIGATING THE CLINICAL AND COST BURDEN OF PRIMARY SJÖGREN'S SYNDROME: A DESCRIPTIVE ANALYSIS FROM A US ADMINISTRATIVE CLAIMS DATABASE}

S. Perera ${ }^{1}$, L. $\mathrm{Ma}^{2}$, R. Punwaney ${ }^{3}, \mathrm{~S}$. Ramachandran ${ }^{4} .{ }^{1}$ GSK, London, United Kingdom; ${ }^{2}$ GSK, Pennsylvania; ${ }^{3}$ GSK, New York; ${ }^{4}$ GSK, King of Prussia, United States

Background: There is limited information available describing the disease burden of primary Sjögren's Syndrome (pSS).

Objectives: This retrospective cohort study aimed to provide a descriptive analysis of the clinical characteristics and disease burden of patients with pSS and describe their healthcare resource use and associated costs.

Methods: This retrospective cohort study (HO-15-16077) was conducted in the US Truven MarketScan ${ }^{\circledR}$ Commercial database. This database reports descriptive data from employees and their families, as covered by employer-sponsored private health insurance. The primary cohort comprised patients with $\geq 1$ diagnosis of sicca syndrome prior to 1 January 2013, and continued enrolment for $>24$ months ( 1 January 2012 to 31 December 2013). Patients with any conditions mimicking sicca symptoms not due to SS were excluded, as were those with connective tissue disease that may suggest secondary SS. The 1:1 matched comparator population comprised subjects without a previous diagnosis of sicca syndrome (sicca-free). The primary objective was to describe the clinical characteristics and demographics of patients with pSS, including the proportion of patients with glandular versus extra-glandular disease. The secondary objective was to describe their healthcare costs and resource utilisation compared with sicca-free subjects.

Results: There were 12,717 eligible patients in the primary cohort: the majority $(86 \%)$ were female and the mean age was 51 years. Overall, $61 \%$ of patients had claims associated with extra-glandular disease manifestations. These patients experienced a higher disease burden with $41 \%$ reporting pain, fatigue and/or insomnia, compared with $12 \%$ of patients with glandular disease only who reported pain, fatigue and/or insomnia. Patients in the primary cohort incurred greater annual healthcare service costs (1.6 times greater for all causes and 1.5 times greater for pSS-related causes) and healthcare resource utilisation compared with the sicca-free comparator cohort. The largest difference in SS-related resource utilisation was in the number of office visits (primary cohort: 5.8 visits per patient; comparator cohort: 4.7 visits per patient). Patients with extra-glandular disease also incurred greater average annual costs and used more healthcare resources than patients with glandular disease only. 
Conclusions: Despite treatment, patients with pSS still experience a high disease burden. Here we have provided novel insights into the higher treatment cost and increased healthcare utilisation burden of pSS compared with the sicca-free cohort, in particular for patients with extra-glandular disease manifestations. Acknowledgements: Study funded by GSK. Jennie Frain, PhD, Fishawack Indicia Ltd, UK, provided editorial assistance funded by GSK

Disclosure of Interest: S. Perera Shareholder of: GSK, Employee of: GSK, L. Ma Shareholder of: GSK, Employee of: GSK, R. Punwaney Shareholder of: GSK, Employee of: GSK, S. Ramachandran Shareholder of: GSK, Employee of: GSK DOI: 10.1136/annrheumdis-2017-eular.2352

\section{FRI0764-HPR MORTALITY IN PATIENTS WITH RHEUMATOID ARTHRITIS AND END STAGE RENAL DISEASE}

S. Paudyal ${ }^{1}$, J. Waller ${ }^{2}$, A. Oliver ${ }^{3}$, B. Le ${ }^{3}$, N. Zleik ${ }^{3}$, N.S. Nahman ${ }^{3,4}$, L. Carbone ${ }^{3,4}{ }^{1}$ Internal Medicine, School of Medicine, University of South Carolina, Columbia; ${ }^{2}$ Biostatistics; ${ }^{3}$ Medical College of Georgia, Augusta University; ${ }^{4}$ Charlie Norwood Veterans Affairs Medical Center, Augusta, United States

Background: Cardiovascular related mortality is higher in patients with rheumatoid arthritis (RA) compared to the general population, and accounts for more than half of all deaths in end stage renal disease (ESRD). The prevalence of ESRD is increasing and there are an increasing number of older patients with RA. Our recent study demonstrated approximately $1 \%$ of patients with ESRD have RA. The implications of ESRD on RA relative to the burdens of cardiovascular diseases, cardiovascular and all-cause mortality are not known.

Objectives: To determine whether patients with RA who have ESRD are at increased risk for cardiovascular disease (CVD) events, cardiovascular mortality and all-cause mortality compared to the general population of patients with ESRD. Methods: Retrospective cohort study of adult patients (age 18 and older) with ESRD receiving renal replacement therapy (hemodialysis or peritoneal dialysis) in the United States Renal Data System (USRDS) who initiated dialysis between 2005 and 2008 followed for up to five years. Patients with an ICD-9 diagnostic code for RA on or before the start of dialysis and a $5 \%$ random sample of those without RA were included. Incident cardiovascular events, cardiovascular related mortality and all-cause mortality was determined in those with RA compared to those without RA.

Results: There were 2,824 subjects including 407 with $R A$ and 2,417 without RA included in the analyses. There was no significant difference in the total number of incident CVD events by RA status ( $n=311(76.4 \%$ RA) vs. $n=1936(80.1 \%$ without RA) ( $p=0.09)$. 76 patients with RA (18.7\%) died from a CVD related cause compared to 403 without $R A(16.7 \%),(p=0.32)$. Overall mortality was significantly higher in those with RA ( $n=226(55.5 \%)$ vs. $n=970(40.1 \%)(p<0.01)$. Compared to those without RA, those with RA had a significantly shorter mean time in months from start of dialysis to any incident CVD event (17.5 (12.4)) vs. 21.2 (14.1) $(p<0.01)$, CVD death, (34.2 (12.5) vs.37.9 (12.6) $p=0.02)$, or all-cause mortality (33.1 (13.0) vs. $37.8(12.6)(p<0.01)$. In final adjusted models, RA was associated with an increased risk for both CVD related mortality (aHR=1.23 $(95 \%$ $\mathrm{Cl} 1.05-1.43)$ ) and all-cause mortality (aHR=1.22 (1.05 - 1.42) within five years. Risk factors for CVD and overall mortality included older age, a higher Charlson comorbidity index, tobacco use, needing assistance with ADLs and living in a nursing home. Black race and Hispanic ethnicity was associated with significantly less CVD and all cause-related mortality.

Conclusions: Physicians treating patients with RA and ESRD should be aware that these patients are at increased risk for cardiovascular related mortality and all-cause mortality compared with the general population of ESRD patients. Patients with ESRD and RA at higher risk for mortality can be identified by both demographic risk factors as well as overall health status.

Acknowledgements: Funding for this work was supported by the Translational Research Program (TRP) at the Medical College of Georgia and the Medical and Graduate Student Preceptorship award from the Rheumatology Research Foundation, Atlanta, Georgia.

Disclosure of Interest: None declared

DOI: 10.1136/annrheumdis-2017-eular.1152

\section{FRI0765-HPR PREVALANCE OF SARCOPENIA IN ELDERLY WITH OSTEOARTHRITIS OF LARGE JOINTS}

Y. Safonova ${ }^{1}$, V. Tsurko ${ }^{2} .{ }^{1}$ Northwestern State Medical University named after II Mechnikov, Department of gerontology, Geriatrics and Nursing, St. Petersburg;

${ }^{2}$ First Moscow State University named after IM Setchenov Department of Hematology and Geriatrics, Moscow, Russian Federation

Background: Lean muscle mass and strength decline starting approximately at 40 years of age to become $25 \%$ of body weight at $75-80$ years old [1]. Within the existing literature, sarcopenia is a highly prevalent condition in older people. The prevalence of sarcopenia increases considerably with age ranging from $5 \%$ to $13 \%$ in 60 to 70 years, from $11 \%$ to $50 \%$ for the population aged 80 years and older. In older persons, sarcopenia is related to falls and physical disability leading to reduced quality of life [2]. The prevalence of osteoarthritis increases with age so that 30 to $50 \%$ of adults over the age of 65 years suffer from this condition [3]. Age-related factor that contributes includes to the development of OA include a decline in muscle strength. People with lower extremity OA had a two to five times increased incidence of falls than age-matched healthy controls [4].

Objectives: Conduct analysis of condition of muscle strength and muscle functioning in older persons with osteoarthritis.

Methods: Prospective study of 159 patients aged $74 \pm 13,3$ years was held. Condition of sarcopenia was estimated by lean body mass (LBM) in accordance with criteria of sarcopenia EWGSOP. Muscle strength was estimated by a hand dynamometer and muscle functioning was estimated on the basis of SPPB tests. Amount of pain was estimated by VAS.

Results: Sarcopenia was revealed in $31,45 \%$ of older persons with ostearthritis. Cases of falls were observed in $28,30 \%(95 \% \mathrm{Cl} 21,5-36,0)$ in patients with osteoartritis with sarcopenia (average number of falls $-1,93$ ) and in $16,98 \%$ of patients without sarcopenia $(95 \% \mathrm{Cl} 11,5-23,7)$ (average number of falls 0,48 ). Level of pain in patients with osteoartritis with sarcopenia amounted 3,16 points, in patients without sarcopenia $-3,49$ points $(p>0,05)$. Muscle strength in patients with sarcopenia was $14,36 \mathrm{~kg}$, in patients without sarcopenia was significantly higher $-18,53 \mathrm{~kg}(p<0,05)$. Common point of SPPB tests in patients with sarcopenia was 6,9 , in patients without sarcopenia significantly higher $-7,85$ $(p<0,05)$

Conclusions: Patients with sarcopenia in the presence of osteoarthritis were observed to have significant decrease of muscle strength and muscle functioning, increase of frequency of falls which raises risk of repeated falls and their frequency, and consequently, deteriorates condition of musculoskeletal system in older persons.

References:

[1] Ferrucci L., Baroni M., Ranchelli A. et al. Interaction Between Bone and Muscle in Older Persons with Mobility Limitations. Curr Pharm Des. 2014; 20(19): 3178-3197.

[2] Morley JE. Sarcopenia: diagnosis and treatment. J Nutr Health Aging. 2008; 12:452-456.

[3] Felson DT. Risk factors for osteoarthritis: understanding joint vulnerability. Clinical orthopaedics and related research 2004;(427 Suppl):S16-21.

[4] Hoops ML, Rosenblatt NJ, Hurt CP, Crenshaw J, Grabiner MD. Does lower extremity osteoarthritis exacerbate risk factors for falls in older adults? Womens Health (Lond Engl). 2012;8(6):685-96.

Disclosure of Interest: None declared

DOI: 10.1136/annrheumdis-2017-eular.3097

\section{FRIDAY, 16 JUNE 2017 \\ HPR professional education, training and competencies}

\section{FRI0766-HPR DOWN'S ARTHROPATHY - CLINICAL AND RADIOLOGICAL FEATURES OF ARTHRITIS IN CHILDREN WITH TRISOMY 21}

\section{Foley, E.J. MacDermott, O.G. Killeen. OLCHC, Dublin, Ireland}

Background: Down's Arthropathy (DA) was first reported in the literature in 1984. Crude estimates suggest higher incidence and prevalence rates of DA compared with Juvenile Idiopathic Arthritis (JIA), (JIA prevalence 1/1000, estimated DA prevalence $8.7 / 1000$ ). Despite this fact, there remains a paucity of data on this condition. DA is rarely recognised at onset, \& remains under-diagnosed. As a direct consequence children with DA are presenting with significant joint damage and disability at diagnosis.

Objectives: Perform a musculoskeletal examination on children with Trisomy 21 (T21) aged $0-20$ years

Methods: Children with T21 were invited to attend a screening clinic. Screening involved completion of a health questionnaire \& a comprehensive musculoskeletal examination. DA cases detected were investigated \& managed as per normal clinical practice. Data on a convenience sample of 33 newly diagnosed children with JIA was collected to create a comparison group.

Results: 503 children with T21 have been screened for DA, 22 new cases have been diagnosed. All of these children had poor language skills or were non-verbal. Only $11 \%$ of the parents suspected that their child may have arthritis prior to attending our screening clinics, and this was only after reading our recruitment literature. In total, we now have 33 children attending our centre with DA (combining cases attending pre-dating the start date of the study). This suggests the prevalence of DA in Ireland is $18-21 / 1000$.

The majority of children presented with a polyarticular pattern of disease. No cases of uveitis have been observed to date. $88 \%$ of the DA cohort had small joint involvement of the hands, significantly higher than that observed in the JIA comparison group. Erosive changes were reported on X-Ray in $29.2 \%$ of the DA cohort (9.5\% in the JIA Cohort). Methotrexate-associated nausea was a significant barrier to treatment with this DMARD in DA. There was a significant delay in diagnosis of DA, 1.7 years $v 0.7$ years in the JIA cohort.

Conclusions: Children with T21 are at increased risk of developing arthritis. There is a lack of awareness of this risk among health care professionals \& the general public at large. This almost certainly contributes to poor recognition of the disease and a delay in diagnosis. The predominant pattern of disease is polyarticular small joint arthritis. Treatment with standard protocols used in JIA 